03

\title{
Особенности распространения и поглощения электромагнитных сигналов в периодических структурах из проводящих и диэлектрических слоев
}

\author{
(C) А.В. Богацкая ${ }^{1,2,3}$, Н.В. Кленов ${ }^{1}$, П.М. Никифорова ${ }^{1,4}$, А.М. Попов ${ }^{1,2}$, А.Е. Щеголев ${ }^{3,4}$ \\ ${ }^{1}$ Московский государственный университет им. М.В. Ломоносова, фризический фракультет, \\ 119991 Москва, Россия \\ 2 Физический институт им. П.Н. Лебедева РАН, \\ 119991 Москва, Россия \\ ${ }^{3}$ Московский технический университет связи и информатики, \\ 111024 Москва, Россия \\ ${ }^{4}$ Научно-исследовательский институт ядерной фризики им. Д.В. Скобельцына Московского государственного \\ университета им. М.В. Ломоносова, \\ 119991 Москва, Россия \\ e-mail: annabogatskaya@gmail.com
}

Поступила в редакцию 20.12.2021 г.

В окончательной редакции 20.12.2021 г.

Принята к публикации 30.12.2021 г.

Обсуждены особенности различных режимов прохождения электромагнитных сигналов через гетероструктуры, образованные последовательностью проводящих и непроводящих слоев. Выявлены разные режимы прохождения при различных соотношениях между частотой распространяющегося излучения и транспортной частотой рассеяния электронов в проводящих слоях. Проанализирована возможность повышения эффективности детектирования или фильтрации электромагнитного излучения в широком диапазоне частот (от субтерагерцового до дальнего ИК) в таких структурах.

Ключевые слова: распространение электромагнитных волн в гетероструктурах, резонансное туннелирование, детекторы ТГц-ИК диапазона, болометры.

DOI: $10.21883 /$ OS.2022.04.52259.48-21

\section{Введение}

Существующие детекторы ТГц-ИК диапазона можно разделить на две группы по принципу работы: фотонные и тепловые [1-8]. В фотонных детекторах излучение поглощается внутри материала путем внутреннего фотоэффекта, т.е. непосредственного взаимодействия фотонов с носителями заряда. Такие детекторы в отличие от тепловых обладают селективной чувствительностью по частоте, для них характерны высокое быстродействие и высокое отношение сигнал/шум. Однако терагерцовое (ТГц) излучение характеризуется малой энергией фотона, поэтому фотонные ТГц устройства с квантовыми переходами могут работать только при низких температурах, что крайне неудобно для практического использования. Принцип действия тепловых детекторов основан на превращении падающего излучения в тепло с последующим преобразованием теплового потока в электрический сигнал. К тепловым детекторам относятся различные микроболометры (металлические, полупроводниковые). Достоинствами таких детекторов являются простота конструкции и отсутствие необходимости в криогенном охлаждении, что значительно снижает энергозатраты при функционировании и стоимость приборов. Недостатком болометрических детекторов ТГц диапазона является низкая чувствительность. В работах [9-11] была исследована возможность увеличения чувствительности болометрических приемников для случаев квазимонохроматического и широкополосного ТГц излучения. Как отмечалось в [9], эффективность болометра можно существенно увеличить, если за поглощающим чувствительным элементом расположить диэлектрический слой, играющий роль электромагнитного резонатора и обеспечивающий резонансное прохождение электромагнитных волн через проводящий слой со сверхкритической концентрацией электронов $[12,13]$. Было показано, что такое резонансное прохождение сопровождается эффективным поглощением энергии электромагнитной волны в проводящем слое на той же частоте. Возможность увеличить спектральную ширину детектирования посредством замены одного диэлектрического слоя гетероструктурой из периодической последовательности проводящих и диэлектрических слоев была рассмотрена в $[10,11]$. В $[10,11]$ было показано, что в такой гетероструктуре аналогично разрешенным зонам фотонного кристалла будут образовываться зоны, в пределах которых также возможно увеличение поглощения. Но в отличие от фотонного кристалла зоны прохождения и поглощения по-разному формируются в исследуемой гетероструктуре. 


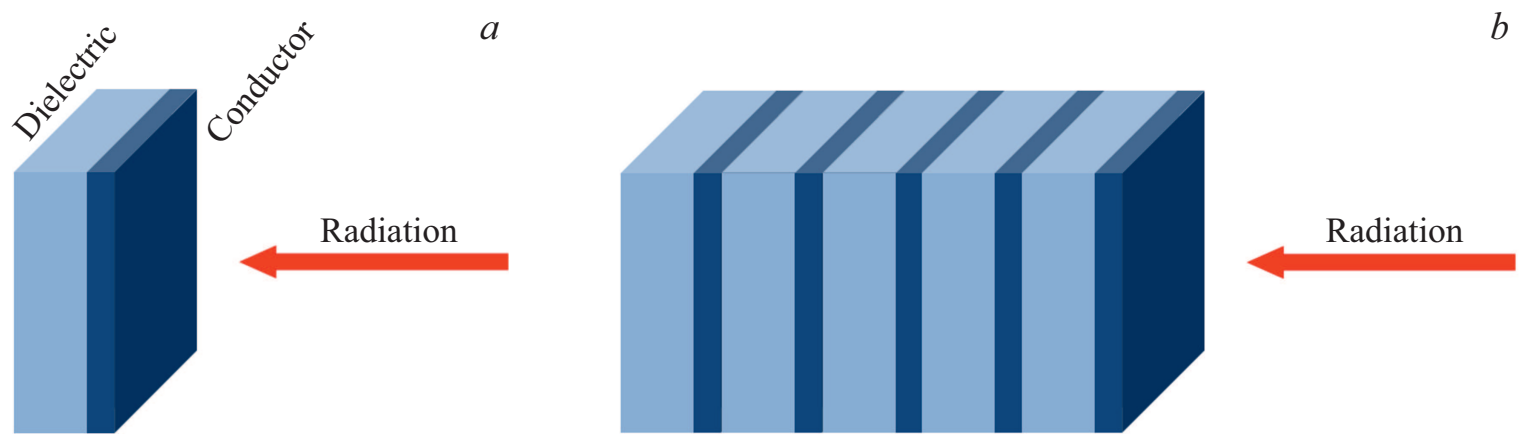

Рис. 1. Гетероструктура из последовательности $N$ проводящих и непроводящих слоев на основе легированного и нелегированного арсенида галлия: $(a) N=1,(b) N=5$.

В настоящей работе рассматриваются особенности, связанные с различными режимами прохождения электромагнитных сигналов через гетероструктуру в зависимости от соотношения между частотой детектируемого излучения и транспортной частотой рассеяния носителей заряда в проводящих слоях, и обсуждается возможность создания как детекторов, так и фильтров электромагнитного излучения в широком диапазоне частот (от субтерагерцового до дальнего ИК) на основе таких структур. Характерный вид исследуемых ниже структур в случае различного числа проводящих и непроводящих слоев представлен на рис. 1 .

\section{Постановка задачи. Моделирование распространения электромагнитной волны в периодической гетероструктуре}

Пространственная структура электрического поля монохроматической волны с частотой $\omega$, падающей нормально на пространственно неоднородную среду с диэлектрической проницаемостью $\varepsilon_{\omega}(z)(z$ направлено по нормали к поверхности), описывается уравнением Гельмгольца для напряженности электрического поля $E(z)$ :

$$
\frac{d^{2} E(z)}{d z^{2}}+\frac{\omega^{2}}{c^{2}} \varepsilon_{\omega}(z) E(z)=0,
$$

где, согласно [9-11], диэлектрическая проницаемость может быть представлена в виде

$$
\varepsilon_{\omega}=\left\{\begin{array}{cc}
\varepsilon_{0}, & 0<z \leq a, \\
\varepsilon_{0}-\frac{\omega_{p}^{2}}{\omega^{2}+v^{2}}+i \frac{\omega_{p}^{2}}{\left(\omega^{2}+v^{2}\right) \omega}, \quad a \leq z \leq a+b, \\
\text { в проводящем слое }, \\
\varepsilon_{\text {air }}, \quad z \geq a+b, \\
\text { вне периодической структуры. }
\end{array}\right.
$$

Здесь $a$ и $b$ - толщины диэлектрического и проводящего слоев соответственно, $\varepsilon_{0}$ - диэлектрическая проницаемость нелегированного слоя. В проводящих слоях для диэлектрической проницаемости мы использовали выражение из теории Друде, $\omega_{p}^{2}=4 \pi e^{2} n_{e} / m^{*}-$ плазменная частота, определяемая величиной $n_{e}\left(n_{e}-\right.$ концентрация носителей $n$-типа), $m^{*}$ - эффективная масса носителей $n$-типа, $v$ - эффективная (транспортная) частота столкновений, $\varepsilon_{\text {air }} \approx 1-$ диэлектрическая проницаемость воздуха. При этом в дальнейшем полагается, что в плоскости $z=0$ находится идеально отражающая поверхность. Известно, что типичные значения транспортных частот $v$ наиболее часто используемых полупроводников (GaAs, $\mathrm{Ge}, \mathrm{Si}$ ) лежат в пределах от $8 \cdot 10^{11}$ до $3 \cdot 10^{12} \mathrm{~s}^{-1}$, поэтому в случае ИК излучения выполняется неравенство $v \ll \omega \ll \omega_{p}$, в то время как в случае субтерагерцогого излучения выполняется обратный случай $\omega<v \ll \omega_{p}$. Как будет показано в дальнейшем, задача имеет существенно разные решения в зависимости от соотношения между транспортной частотой $v$ и частотой распространяющегося излучения $\omega$ (при этом мы полагаем, что $\left.\omega, v \ll \omega_{p}\right)$.

Для ИК диапазона частот в нулевом приближении по малому параметру $v / \omega_{p}$ диэлектрическая проницаемость проводящего слоя может быть записана как $\varepsilon_{\omega} \approx \varepsilon_{0}-\frac{\omega_{p}^{2}}{\omega^{2}}$. Тогда уравнение (1) может быть переписано в виде

$$
\frac{d^{2} E(z)}{d z^{2}}+\frac{\omega^{2}}{c^{2}}\left(\varepsilon_{0}-\frac{\omega_{p}^{2}}{\omega^{2}}\right) E(z)=0 .
$$

Нетрудно заметить, что это уравнение математически аналогично стационарному уравнению Шредингера для частицы массы $m$ в потенциале $V(z)$ :

$$
-\frac{\hbar^{2}}{2 m} \frac{d^{2} \psi(z)}{d z^{2}}+V(z) \psi(z)=\xi \psi(z)
$$

где $\psi(z)$ - волновая функция стационарного состояния с энергией $\xi$. Таким образом, наличие диэлектрического слоя (резонатора), находящегося за проводящим плазменным покрытием, с точки зрения квантовой механики аналогично наличию потенциальной ямы, находящейся 
за потенциальным барьером. Тогда решение уравнения (3), описывающее проникновение электромагнитного поля через область со сверхкритической концентрацией электронов, можно интерпретировать в рамках аналогии с квантовой механикой как туннелирование частиц через потенциальный барьер. При этом при совпадении частоты падающего поля с частотой собственных мод резонатора будет наблюдаться эффект резонасного туннелирования поля через „плазменный барьер“, что приведет к эффективному заполнению диэлектрического резонатора электромагнитным полем.

Для расчета поглощения энергии поля в структуре необходимо учесть наличие мнимой части диэлектрической проницаемости:

$$
\varepsilon_{\omega} \approx \varepsilon_{0}-\frac{\omega_{p}^{2}}{\omega^{2}}+i \frac{\omega_{p}^{2} v}{\omega^{3}}
$$

Ниже в роли проводника рассматриваются слои легированного GaAs, а в качестве диэлектрика - слои нелегированного GaAs. При этом уровень легирования выбирается так, чтобы частота падающего поля была ниже критической, а толщина скин-слоя $\delta$ была порядка толщины проводящего слоя. Для рассматриваемого случая $v \ll \omega \ll \omega_{p}$ толщину скин-слоя можно оценить по формуле $\delta \approx c / \omega_{p}$, что накладывает ограничение на толщину легированного полупроводника.

Эффективность проникновения электрического поля внутрь диэлектрического резонатора и „плазменного барьера“" удобно характеризовать фактором заполнения [13], определяемым как

$$
F_{1,2}(\omega)=\max \left\{\frac{\left|E_{1(2)}\right|^{2}}{\left|E_{0}\right|^{2}}\right\},
$$

где $E_{1(2)}-$ максимальные значения напряженности электрического поля волны, прошедшей в область диэлектрического (проводящего) слоя волны, полученные из численного решения уравнения Гельмгольца (1), a $E_{0}$ - напряженность электрического поля падающей волны.

Другая ситуация возникает в низкочастотных полях, когда $\omega \ll v \ll \omega_{p}$. В этом случае в проводящем слое мнимая часть диэлектрической проницаемости становится главным слагаемым, и в приближении низких частот можно записать $\varepsilon_{\omega} \approx i \frac{\omega_{p}^{2}}{v \omega}$. Уравнение Гельмгольца в проводящем слое принимает вид

$$
\frac{d^{2} E(z)}{d z^{2}}+i \frac{\omega \omega_{p}^{2}}{v c^{2}} E(z)=0
$$

Очевидно, что по своей структуре это уравнение сильно отличается от уравнения (3) для случая высокочастотного поля. Поэтому естественно ожидать другой характер распространения электромагнитной волны через гетероструктуру.

\section{Результаты моделирования. Формирование зон прохождения и поглощения электромагнитной волны в периодической гетероструктуре из легированных и нелегированных слоев GaAs}

На рис. 2, $а$ изображены факторы заполнения для диэлектрического слоя $F_{1}$ и для проводящего слоя $F_{2}$ в зависимости от частоты излучения, полученные при численном интегрировании уравнений (1), (2) в случае $\omega \gg \mu$. Также на рис. 2, $a$ изображена кривая поглощения $\eta$ в зависимости от частоты излучения. Поглощение рассчитывалось нами по следующей формуле [10]:

$$
\eta(\omega)=\frac{\omega_{p}^{2} v}{\omega^{2}+v^{2}} \frac{\int E^{2}(z) d z}{c E_{0}^{2}} .
$$

Интеграл в (8) берется по области проводящего слоя.

Как видно, эффективное туннелирование электромагнитной волны внутрь резонатора на определенных частотах сопровождается существенным проникновением поля в проводящий слой на этих же частотах, что ведет к возрастанию доли поглощаемой энергии. При этом наличие квадрата частоты в знаменателе (8) приводит к тому, что максимальное поглощение наблюдается при минимальном значении резонансной частоты. Положение резонансных частот можно определить из условия:

$$
\omega_{n} \approx \frac{\pi c}{a \sqrt{\varepsilon_{0}}} n
$$

где $a$ - толщина диэлектрического слоя (нелегированного GaAs), $n$ - номер резонанса. В рассматриваемых условиях такая структура обеспечивает поглощение порядка 80-90\% энергии падающего излучения на частотах, определяемых выражением (9). В дальнейшем именно условие (9) лежало в основе выбора толщины диэлектрических слоев для анализа прохождения электромагнитной волны в режимах $v \ll \omega \ll \omega_{p}$ и $\omega \ll v \ll \omega_{p}$.

Качественно другая структура поля в системе возникает в низкочастотном случае $\omega \ll v \ll \omega_{p}$. Если положить толщину скин-слоя, определяемую в этом случае выражением $\delta \approx \frac{c}{\omega_{p}} \sqrt{\frac{2 v}{\omega}}$, порядка толщины проводящего слоя, проникновение поля в проводящую и непроводящую область структуры оказывается незначительным, из-за чего поглощение сигнала также будет пренебрежимо малым. Поэтому далее мы рассматриваем случай, когда скин-слой $\delta$ много больше толщины проводящего слоя. Для такой ситуации на рис. 2, $b$ изображены факторы заполнения для диэлектрического слоя $F_{1}$ и для проводящего слоя $F_{2}$, а также кривая поглощения $\eta$. Видно, что в данном случае эффективное проникновение электрического поля в диэлектрический резонатор не сопровождается возрастанием напряженности поля внутри проводящего слоя (рис. 2, b). Напротив, 
$a$
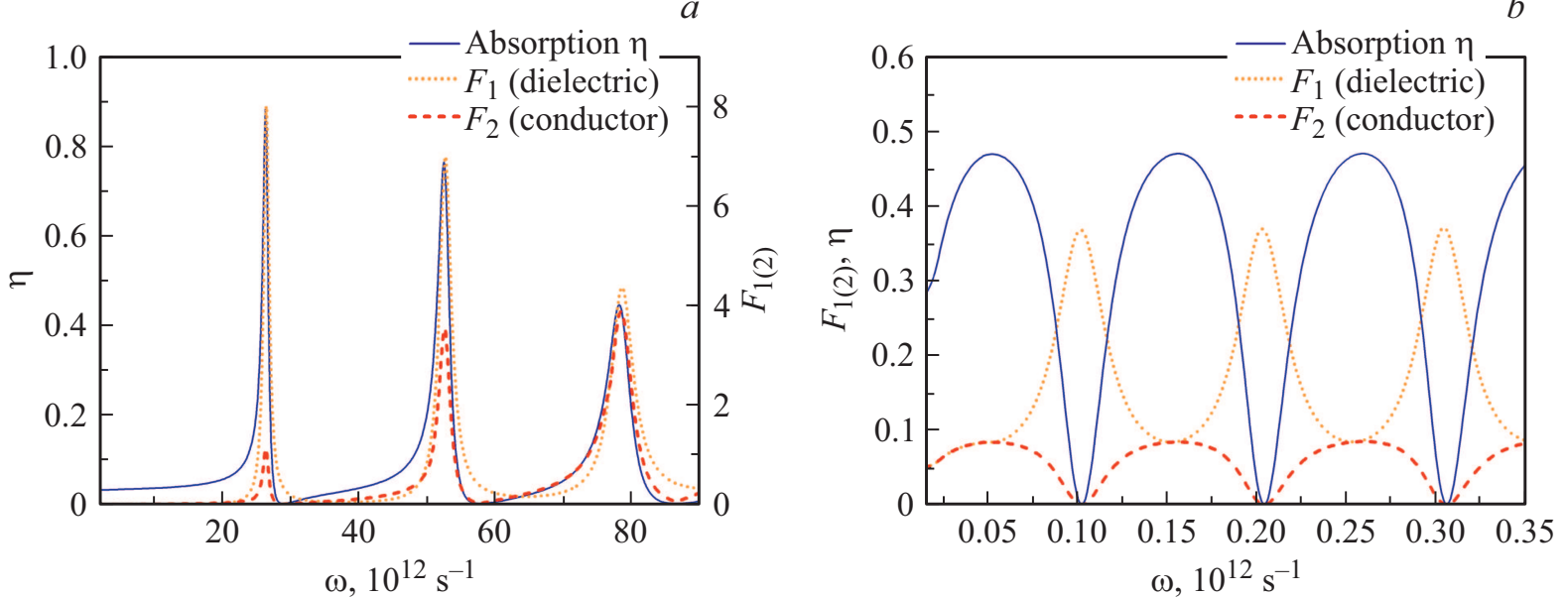

Рис. 2. Поглощение $\eta$ и факторы заполнения $F_{1,2}$ в зависимости от частоты падающего излучения. Параметры структуры: (a) толщина диэлектрического слоя $a=9.5 \mu \mathrm{m}$, толщина проводящего слоя $b=1 \mu \mathrm{m}$, плазменная частота $\omega_{p}=3 \cdot 10^{14} \mathrm{~s}^{-1}$, транспортная частота в GaAs $v=3.09 \cdot 10^{12} \mathrm{~s}^{-1} ;(b) a=2800 \mu \mathrm{m}, b=1 \mu \mathrm{m}, \omega_{p}=7.6 \cdot 10^{13} \mathrm{~s}^{-1}, v=3.09 \cdot 10^{12} \mathrm{~s}^{-1}$.
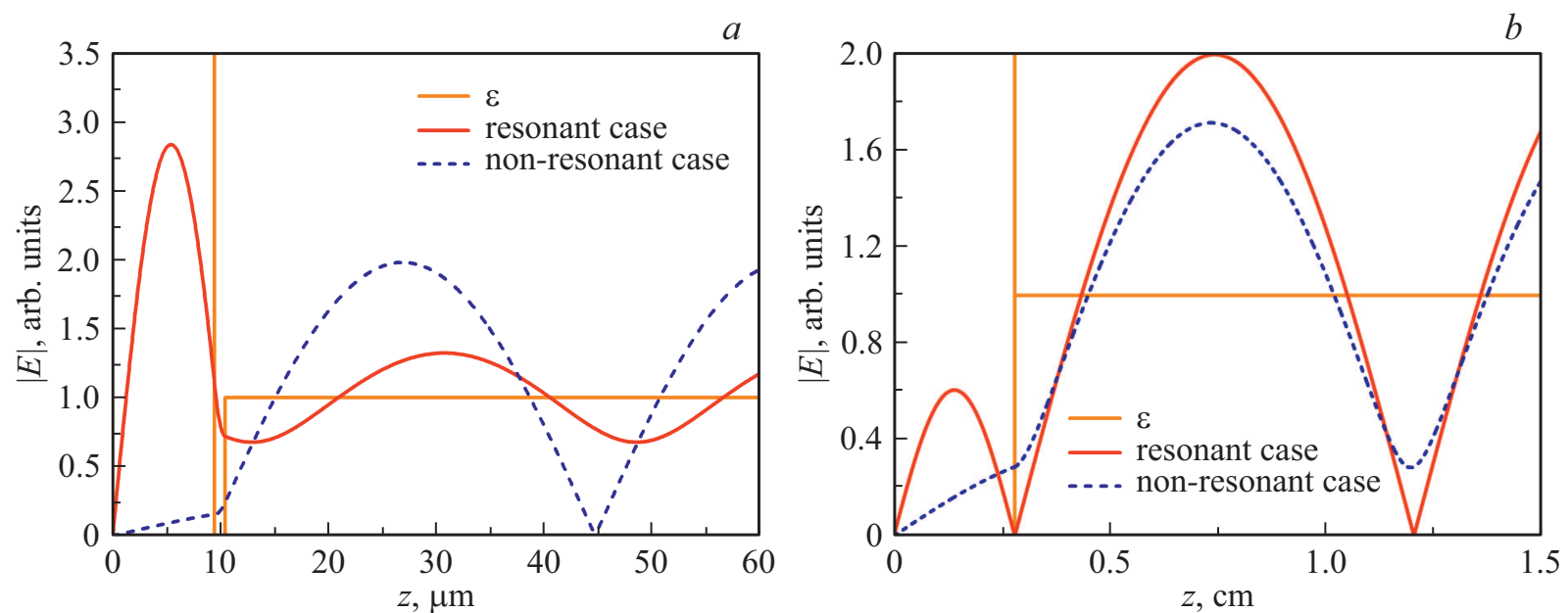

Рис. 3. Пространственное распределение напряженности электрического поля. Параметры структуры: $(a)$ толщина диэлектрического слоя $a=9.5 \mu \mathrm{m}$, толщина проводящего слоя $b=1 \mu \mathrm{m}$, плазменная частота $\omega_{p}=3 \cdot 10^{14} \mathrm{~s}^{-1}$, транспортная частота в $\mathrm{GaAs}$ $v=3.09 \cdot 10^{12} \mathrm{~s}^{-1} ;(b) a=2800 \mu \mathrm{m}, b=1 \mu \mathrm{m}, \omega_{p}=7.6 \cdot 10^{13} \mathrm{~s}^{-1}, v=3.09 \cdot 10^{12} \mathrm{~s}^{-1}$.

на частотах, соответствующих частотам собственных мод резонатора, наблюдаются минимумы поглощения.

Для объяснения различия резонансных кривых в низко- и высокочастотном случаях рассмотрим пространственное распределение модуля напряженности электрического поля в исследуемой структуре (рис. 3). В высокочастотном случае в условиях совпадения частоты поля с частотой одной из мод резонатора (график построен для частоты поля, совпадающей с частотой первой моды) поле хорошо проникает в диэлектрический слой, что сопровождается эффективным поглощением в проводящем слое. Штриховой кривой на графике представлено распределение поля в том случае, когда в качестве диэлектрика под проводящим слоем взят материал с $\varepsilon=1$. Частоты для обоих представленных на графике полей одинаковы, но в случае штриховой кривой эта частота уже не является резонансной. Видно, что в отсутствие резонанса проникновение поля внутрь проводящей области существенно меньше.

На рис. 3, $b$ представлено пространственное распределение модуля напряженности электрического поля для случая $\omega \ll v \ll \omega_{p}$. В резонансном случае поле также эффективно заполняет резонатор. При этом распределение поля таково, что, если на ширине диэлектрика укладывается ровно половина длины волны, в проводящем слое образуется узел, и поскольку проводящий слой достаточно тонкий (по сравнению с длиной волны), поглощение для резонансных частот минимально. Как и прежде, штриховой линией на графике представлено распределение поля в том случае, когда под проводящим слоем находится диэлектрик с $\varepsilon=1$. В этом случае поле хуже заполняет резонатор, но лучше проникает 
$a$

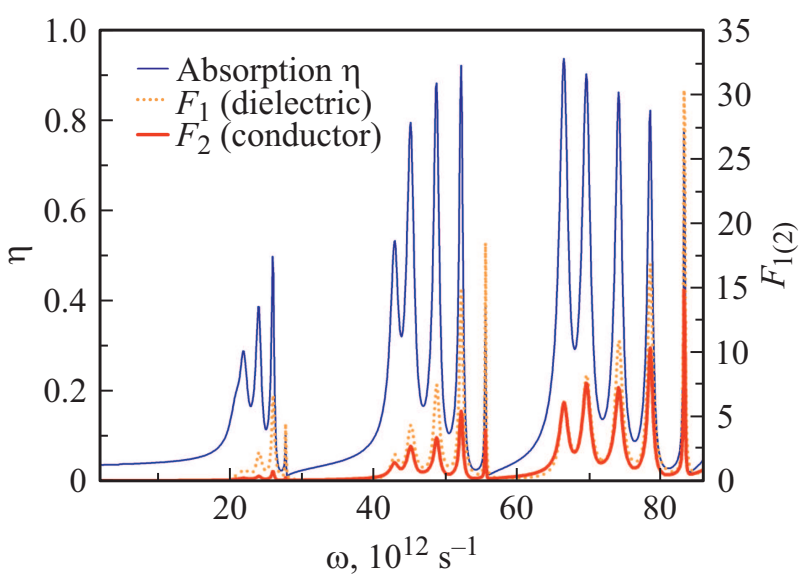

$b$

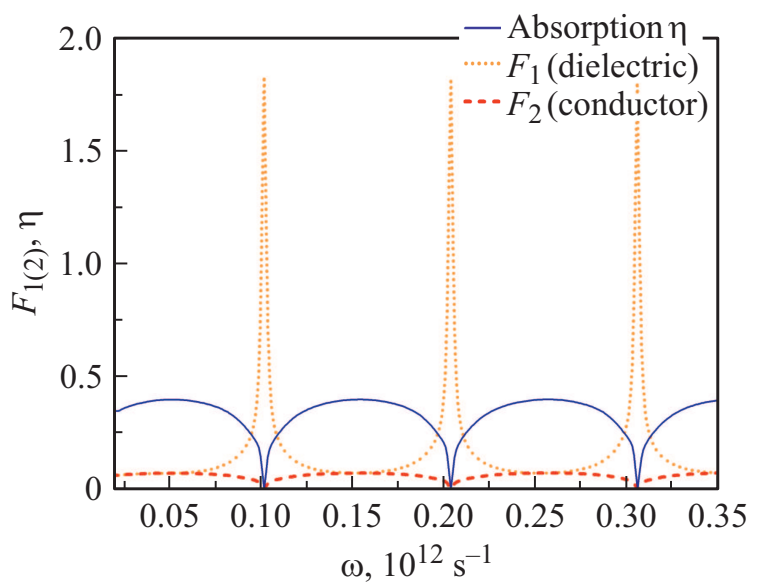

Рис. 4. Поглощение $\eta$ и факторы заполнения $F_{1,2}$ в зависимости от частоты падающего излучения. Параметры структуры: (a) толщина диэлектрического слоя $a=9.5 \mu \mathrm{m}$, толщина проводящего слоя $b=1 \mu \mathrm{m}$, плазменная частота $\omega_{p}=3 \cdot 10^{14} \mathrm{~s}^{-1}$, транспортная частота в GaAs $v=3.09 \cdot 10^{12} \mathrm{~s}^{-1} ;(b) a=2800 \mu \mathrm{m}, b=1 \mu \mathrm{m}, \omega_{p}=7.6 \cdot 10^{13} \mathrm{~s}^{-1}, v=3.09 \cdot 10^{12} \mathrm{~s}^{-1}$. Рассматривается случай пяти проводящих слоев.
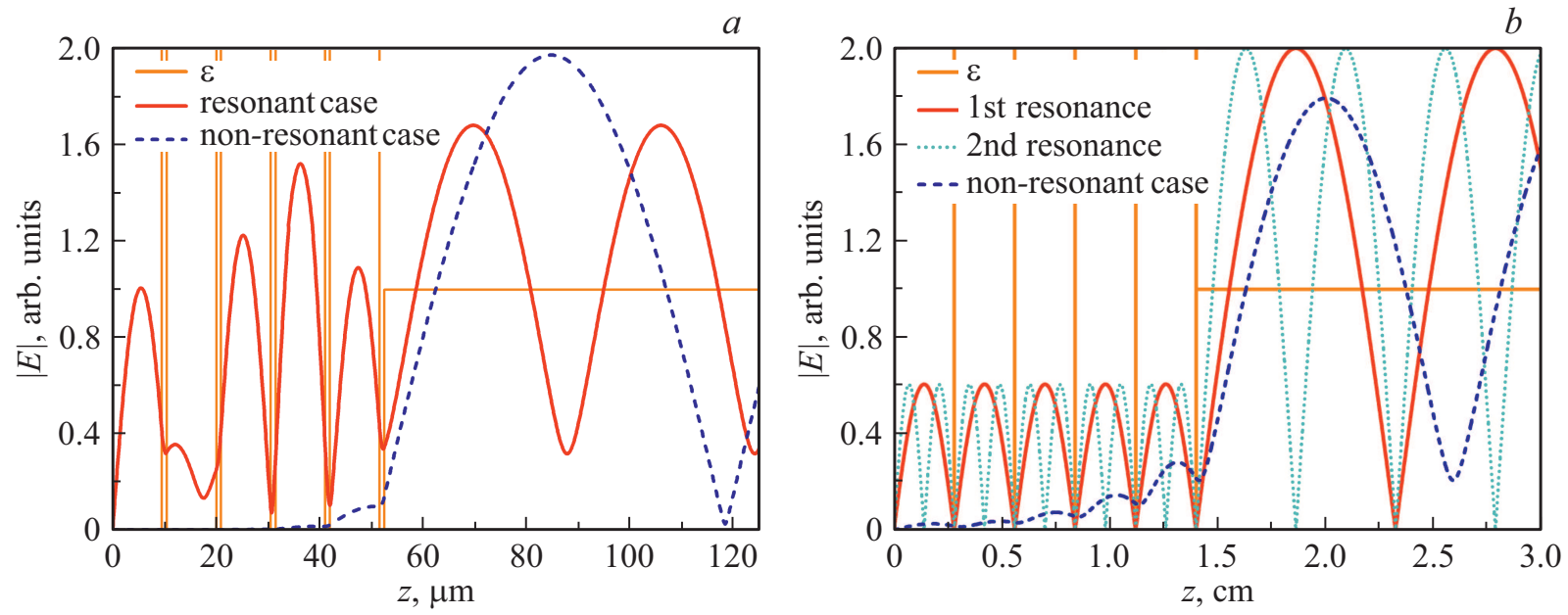

Рис. 5. Пространственное распределение напряженности электрического поля. Параметры структуры: $(a)$ толщина диэлектрического слоя $a=9.5 \mu \mathrm{m}$, толщина проводящего слоя $b=1 \mu \mathrm{m}$, плазменная частота $\omega_{p}=3 \cdot 10^{14} \mathrm{~s}^{-1}$, транспортная частота в GaAs $v=3.09 \cdot 10^{12} \mathrm{~s}^{-1} ;(b) a=2800 \mu \mathrm{m}, b=1 \mu \mathrm{m}, \omega_{p}=7.6 \cdot 10^{13} \mathrm{~s}^{-1}, v=3.09 \cdot 10^{12} \mathrm{~s}^{-1}$.

в проводящий слой. Таким образом, в низкочастотном случае внедрение диэлектрика позади проводящего слоя уменьшает поглощение энергии поля для набора резонансных частот.

Далее мы рассматриваем особенности распространения и поглощения электромагнитных волн в гетероструктурах, состоящих из последовательности пяти проводящих и диэлектрических слоев.

На рис. 4, $a$ приведены факторы заполнения ${ }^{1}$ диэлектрических, $F_{1}$, и проводящих, $F_{2}$, слоев для излучения ИК диапазона $\left(v \ll \omega \ll \omega_{p}\right)$, а также доля поглощения энергии в совокупности проводящих слоев. Видно, что в

\footnotetext{
${ }^{1}$ В данном случае в фактор заполнения (6) входит максимальное значение поля в совокупности диэлектрических (проводящих) слоев.
}

данном случае при увеличении числа слоев формируется зонная структура полевых мод (фотонный кристалл) подобно тому, как формируются разрешенные энергетические зоны в твердом теле. При этом положение разрешенных зон определяется шириной диэлектрического слоя и значением диэлектрической проницаемости (9), а их ширина - связью между соседними диэлектрическими слоями, т. е. вероятностью „туннелирования“ поля через разделяющий диэлектрические слои „потенциальный барьер“ (проводящий слой). Величиной этой связи можно управлять, меняя ширину легированных слоев или степень легирования [10,11]. С увеличением частоты воздействующего излучения растет связь между слоями, что приводит к более эффективному „туннелированию“ поля внутрь структуры и увеличению поглощения. 
В противоположном случае $\left(\omega \ll v \ll \omega_{p}\right)$ численное моделирование показало, что при увеличении числа диэлектрических слоев не происходит формирование зонной структуры полевых мод. Вместо нее возникают узкие уровни, соответствующие прохождению волны на резонансных частотах. На этих же частотах наблюдаются минимумы поглощения (рис. 4,b), причем спектральная ширина уровней уменьшается с увеличением числа слоев.

Иллюстрацией к формированию зон поглощения и прохождения в низко- и высокочастотном случаях являются пространственные распределения напряженности электрического поля для обоих случаев (рис. 5, $a, b$ ). Сравнительный анализ двух графиков показывает, что как в высокочастотном случае (рис. 5, $a$ ), так и для случая низких частот, $\omega \ll v \ll \omega_{p}$ (рис. $\left.5, b\right)$, при определенных значениях частот поле проникает внутрь гетероструктуры и заполняет последовательность диэлектрических резонаторов. Однако при этом поле внутри проводящих слоев оказывается существенно различным: в случае, представленном на рис. 4, $a$, заполнение полем диэлектрических резонаторов сопровождается увеличением поглощения в проводящих слоях (см. сплошную кривую). Наоборот, в случае, представленном на рис. $4, b$, для частот, совпадающих с собственными модами резонатора, поле практически не проникает в проводящие слои, и именно на этих резонансных частотах формируются минимумы зависимости поглощаемой энергии от частоты излучения.

\section{Заключение}

Таким образом, показано, что характер распространения и поглощения электромагнитных волн в гетероструктурах на основе последовательности проводящих и диэлектрических слоев сильно различается для двух режимов, определяемых соотношением частоты поля и транспортной частоты рассения носителей заряда в проводящих слоях: $v \ll \omega$ (режим высоких частот) и $v \gg \omega$ (режим низких частот). Показано, что увеличение доли поглощаемой энергии падающей волны при совпадении ее частоты с частотой одной из мод диэлектрического резонатора (или попадании ее в разрешенную зону в случае структуры из последовательности нескольких диэлектрических и проводящих слоев) происходит только в том случае, когда частота падающего излучения больше транспортной частоты рассеяния носителей заряда в проводящих слоях. Для случая $v \gg \omega$, напротив, наблюдается минимум поглощения на частотах электромагнитных волн, соответствующих их резонансному прохождению. Увеличение количества слоев в такой гетероструктуре ведет к сужению полос резонансного прохождения сигналов. С точки зрения практических приложений использование таких структур для целей увеличения эффективности болометрического детектирования возможно в высокочастотном режиме. Режим низких частот предполагает использование таких гетероструктур в качестве фильтра излучения, что может быть актуально для ряда устройств интегральной фотоники [14-16].

\section{Финансирование работы}

П.М. Никифорова выражает благодарность за поддержку Фонду развития теоретической физики и математики „БАЗИС“ (проект 21-2-1-15-1). А.В. Богацкая благодарит за поддержку фонд стипендий Президента РФ (СП-3120.2022.3). Н.В. Кленов является победителем Конкурса на предоставление грантов преподавателям магистратуры 2020/2021 г. благотворительной программы „Стипендиальная программа Владимира Потанина“. Работа выполнена при поддержке Научнообразовательной школы „Фотоника и квантовые технологии. Цифровая медицина“.

\section{Конфоликт интересов}

Авторы заявляют, что у них нет конфликта интересов.

\section{Список литературы}

[1] Д.С. Копылова, Н.Ю. Болдырев, В.Я. Яковлев, Ю.Г. Гладуш, А.Г. Насибулин. Квантовая электроника, 46 (12), 1163 (2016). DOI: 10.1070/QEL16146

[2] S.J. Rezvani, D. Di Gioacchino, C. Gatti, C. Ligi, M.C. Guidi, S. Cibella, M. Fretto, N. Poccia, S. Lupi, A. Marcelli. Condens. Matter. 5 (2), 33 (2020). DOI: 10.3390/condmat5020033

[3] A.S. Tukmakova, A.V. Asach, A.V. Novotelnova, I.L. Tkhorzhevskiy, N.S. Kablukova, P.S. Demchenko, A.D. Zaitsev, M.K. Khodzitsky. Appl. Sci., 10 (6), 1929 (2020). DOI: 10.3390/app10061929

[4] Z. Liu, Z. Liang, W. Tang, X. Xu. Infrared Physics \& Technology, 105, 103241 (2020). DOI: $10.1016 /$ j.infrared.2020.103241

[5] D.S. Ponomarev, D.V. Lavrukhin, A.E. Yachmenev, R.A. Khabibullin, I.E. Semenikhin, V.V. Vyurkov, M. Ryzhii, T. Otsuji, V. Ryzhii. J. Phys. D., 51, 135101 (2018). DOI: 10.1088/1361-6463/aab11d

[6] P.M. Echternach, B.J. Pepper, T. Reck, C.M. Bradfort. Nat. Astron., 2, 90 (2018). DOI: 10.1038/s41550-017-0294-y

[7] P. Seifert, X. Lu, P. Stepanov, J.R. Retamal, J.N. Moore, K.C. Fong, A. Principi, D.K. Efetov. Nano Lett., 20 (5), 3459 (2020). DOI: 10.1021/acs.nanolett.0c00373

[8] P. Wang, H. Xia, Q. Li, F. Wang, L. Zhang, T. Li, P. Martyniuk, A. Rogalski, W. Hu. Small, 15, 1904396 (2019). DOI: $10.1002 / \mathrm{smll} .201904396$

[9] A.V. Bogatskaya, N.V. Klenov, M.V. Tereshonok, A.M. Popov. Tech. Phys. Lett., 44 (8), 667 (2018). DOI: $10.1134 / \mathrm{S} 1063785018080059$

[10] A.E. Shchegolev, A.M. Popov, A.V. Bogatskaya, P.M. Nikiforova, M.V. Tereshonok, N.V. Klenov. JETP Lett., 111 (7), 371 (2020). DOI: 10.31857/S0370274X20070036

[11] A.V. Bogatskaya, N.V. Klenov, P.M. Nikiforova, A.M. Popov, A.E. Shchegolev. Tech. Phys. Lett., $47(9), 893$ (2021). DOI: $10.1134 / \mathrm{S} 1063785021090029$ 
[12] A.V. Bogatskaya, E.A. Volkova, N.V. Klenov, M.V. Tereshonok, A.M. Popov. IEEE Trans. Antenn. Prop., 68 (6), 4831 (2020). DOI: 10.1109/TAP.2020.2972649

[13] A.V. Bogatskaya, N.V. Klenov, M.V. Tereshonok, S.S. Adjemov, A.M. Popov. J. Phys. D., 51 (18), 185602 (2018). DOI: $10.1088 / 1361-6463 / \mathrm{aab} 756$

[14] A.W. Elshaari, I.E. Zadeh, A. Fognini, M.E. Reimer, D. Dalacu, Ph.J. Poole, V. Zwiller, K.D. Jöns. Nature Comm., 8, 379 (2017). DOI: 10.1038/s41467-017-00486-8

[15] М.А. Ремнев, В.В. Климов. УФН, 188, 169 (2018) [M.A. Remnev, V.V. Klimov. Phys.-Usp., 61, 157 (2018). DOI: $10.367 /$ UFNe.2017.08.038192]

[16] L. Gu, Q. Yuan, Q. Zhao, Y. Ji, Z. Liu, L. Fang, X. Gan, J. Zhao. Journal of Lightwave Technology, 39, 5069 (2021). DOI: $10.1109 / J L T .2021 .3082558 \mathrm{w}$ 